\title{
Acute Cholecystitis Caused by Vancomycin-Resistant Enterococcus faecium in a Morbidly Obese Patient with Multiple Co-Morbidities
}

\author{
Hugo Bonatti, ${ }^{1,2}$ Joshua Tierney, Eriko Kanaya,,3 Zachary Crislip,,4 John Tarpley, and Addison May ${ }^{1}$
}

\begin{abstract}
Background: Vancomycin-resistant Enterococcus faecium (VRE) is a low virulent pathogen. It can cause a variety of infections most commonly in immunosuppressed patients and those with previous exposure to broadspectrum antibiotics.

Case Presentation: A morbidly obese male with multiple comorbidities presented with acute cholecystitis. Because of the high operative risk it was decided to treat the condition with antibiotics and ultrasound-guided percutaneous cholecystostomy tube placement. Cultures of bile revealed VRE and initial therapy consisted of daptomycin. Cultures on day seven after initiation of daptomycin showed continuing growth of VRE and therapy was changed to linezolid and the patient was able to clear VRE from bile and cholecystitis resolved. The tube was removed after six months and interval cholecystectomy was planned after appropriate weight loss. Conclusions: We report the first case of VRE acute cholecystitis in North America. Only two additional cases have been reported thus far, both from Italy. One responded to daptomycin, which is in contrast to our experience. However, similar to the other reported case, our patient had a good response to linezolid, which may be the preferred agent to treat this condition.
\end{abstract}

A CUTE CHOLECYSTITIS is a common surgical condition. Depending on various factors, treatment may include antibiotics and interval cholecystectomy once the infection has resolved or immediate surgery [1]. Patient with various comorbidities such as diabetes mellitus (DM) and immunosuppressed individuals may progress to gangrenous cholecystitis with an increased risk for gallbladder perforation, in which case emergent surgery is indicated. In patients at high operative risk, gallbladder drainage is an option. Most centers prefer percutaneous ultrasound or computed tomography (CT) guided placement of a cholecystostomy tube [2]. Others, however, have used endoscopic retrograde cholangiopancreatogram (ERCP) guided gallbladder stents successfully [3].

Pathogens implicated in acute cholecystitis include Escherichia coli and other gram-negative bacilli, enterococci, streptococci, and various anaerobes including Clostridium perfringens $[4,5]$. Multiple pathogens can be cultured from bile in cases of acute cholecystitis and/or gallbladder empyema [4]. The role of enterococci in biliary infections has been disputed.

Vancomycin-resistant enterococci (VRE) have emerged over the past 20 years as important hospital pathogens [6]. Most commonly VRE are found in patients with significant comorbidities, immunosuppressed individuals, those with prolonged hospitalization, and patients with previous exposure to broad-spectrum antibiotics. Vancomycin-resistant enterococci may cause a variety of infections such as bacteremia and endocarditis, peritonitis, urinary tract infection (UTI), and has also been isolated in cholangitis. In liver transplant recipients with cholangiopathy, VRE is one of the leading pathogens [7]. Linezolid, daptomycin, quinupristindalfopristin, and tigecycline are established treatment options but new agents are being developed [8,9]. Only two cases of

\footnotetext{
${ }^{1}$ Department of Surgery, Vanderbilt University Medical Center, Nashville, Tennesse.

${ }^{2}$ Community Medical Group-Surgical Care, University of Maryland, Easton, Maryland.

${ }^{3}$ Yale University, New Haven, Connecticut.

${ }^{4}$ Department of Surgery, Oklahoma State University Medical Center, Tulsa, Oklahoma.
}

(C) Hugo Bonatti et al. 2016; Published by Mary Ann Liebert, Inc. This Open Access article is distributed under the terms of the Creative Commons License (http://creativecommons.org/licenses/by/4.0), which permits unrestricted use, distribution, and reproduction in any medium, provided the original work is properly credited. 

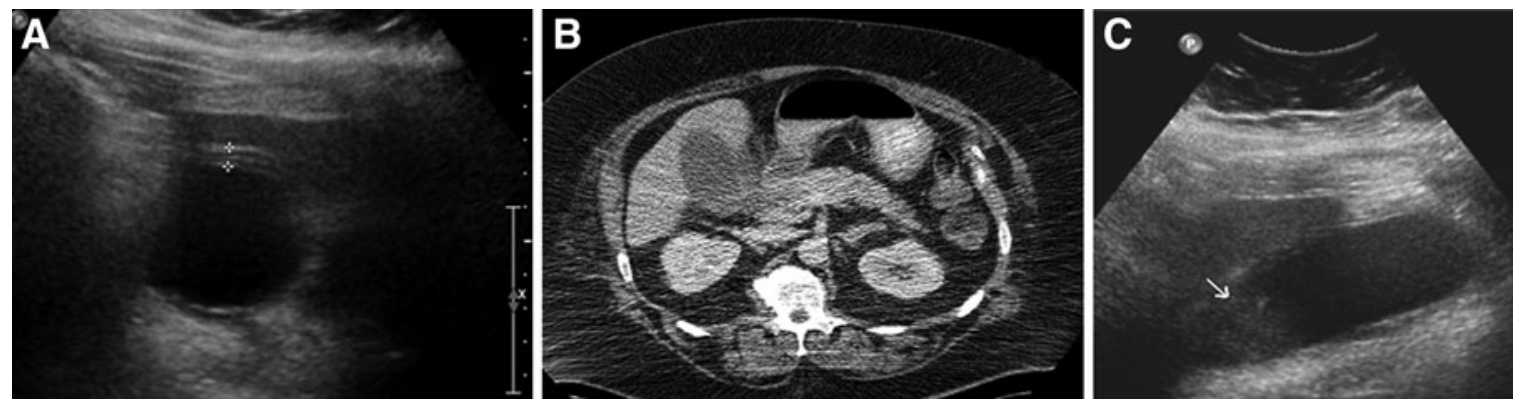

FIG. 1. (A) Ultrasound showing gallbladder wall thickening. (B) Computed tomography scan showing large gallbladder with wall edema. (C) Ultrasound showing distended gallbladder, floating intra-luminal echogenicities, thickening of the gallbladder wall, and a peri-cholecystic fluid.

VRE acute cholecystitis have been published thus far [10,11]; both reports originated from Italy. We herein report the first case of VRE acute cholecystitis in the United States.

\section{Case Presentation}

A 64-year-old African American resident of a nursing care facility was transferred to an outside hospital with a four-day history of increasingly severe right upper quadrant pain and fever. Ultrasound and CT scans were consistent with acute cholecystitis with cholelithiasis (Figs. 1A, 1B, and 1C). The patient had an elevated white blood cell (WBC) count but liver enzymes were normal. He was morbidly obese with a body mass index (BMI) of $55.6 \mathrm{~kg} / \mathrm{m}^{2}$ accompanied by type 2 diabetes mellitus and hypertension. He also had a history of right below-knee amputation, chronic obstructive pulmonary disease (COPD), and obstructive sleep apnea. He had been recently hospitalized for hypercapnic respiratory failure and aspiration pneumonia and had been treated with various antibiotics.

At the hospital, he was initially treated with bowel rest and empiric intravenous piperacillin-tazobactam and vancomycin. Because of a worsening leucocytosis and persistent fever and abdominal pain, he was transferred to our hospital for possible surgical intervention. On physical examination he was febrile and was noted to have tenderness in the right upper quadrant with a positive Murphy sign, but there was no abdominal rebound. His peripheral WBC count on admission was $37.9 \times 10^{3} / \mathrm{mm}^{3}$ and his bilirubin and liver enzymes were within normal range. Ultrasonography of the right upper quadrant showed a distended gallbladder with many floating intra-luminal echogenicities, thickening of the gallbladder wall, and a pericholecystic fluid collection (Fig. 1C). The common bile duct was normal in size.

The patient was hemodynamically stable and because of his operative risk, the decision was made to continue nonoperative management in an intensive care unit (ICU) with bowel rest, intravenous vancomycin, ertapenem, and fluconazole. Computed tomography-guided percutaneous cholecystostomy tube placement was attempted, however, because of his obesity he was unable to fit into our scanner with enough space to perform the intervention. Therefore, on hospital day three, he underwent ultrasound-guided percutaneous cholecystostomy at the bedside and thick turbid bilious fluid was evacuated. Culture of bile grew VRE and daptomycin at a dose of $650 \mathrm{mg} / \mathrm{d}$ was started. However, his respiratory status deteriorated with a $\mathrm{PCO}_{2}$ of $>80 \mathrm{~mm} \mathrm{Hg}$. $\mathrm{He}$ was intubated and placed on a ventilator. The patient's clinical condition slowly improved, the drain output became clearer, and his WBC count trended down. His condition continued to improve and on hospital day 11, the patient was extubated. Repeat culture of bile on hospital day 13 (treatment day seven) remained positive for VRE and thus daptomycin was switched to linezolid, $600 \mathrm{mg}$ orally twice a day. The patient's clinical course continued to improve with no fever and the WBC count normalized. Bile drain output decreased from $300 \mathrm{~mL} / \mathrm{d}$ to $<30 \mathrm{~mL} / \mathrm{d}$. Liver enzymes and total bilirubin all were within normal limits. On hospital day 18 , a
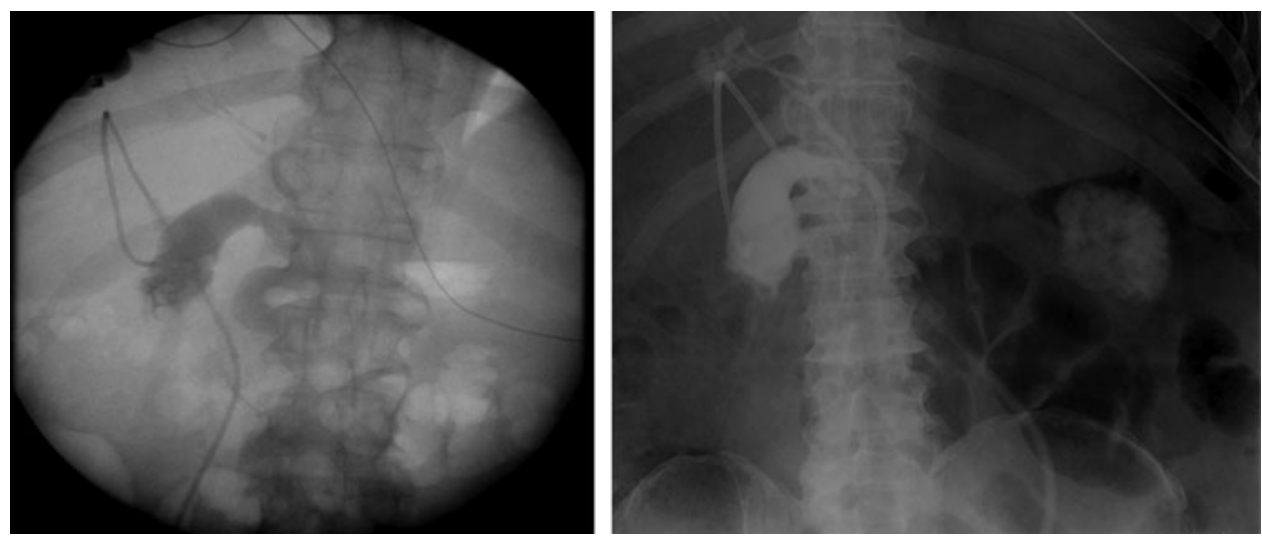

FIG. 2. Cholangiogram through cholecystostomy tube showing correct drain placement, patent cystic duct, and normal common bile duct with unremarkable intrahepatic biliary system. 


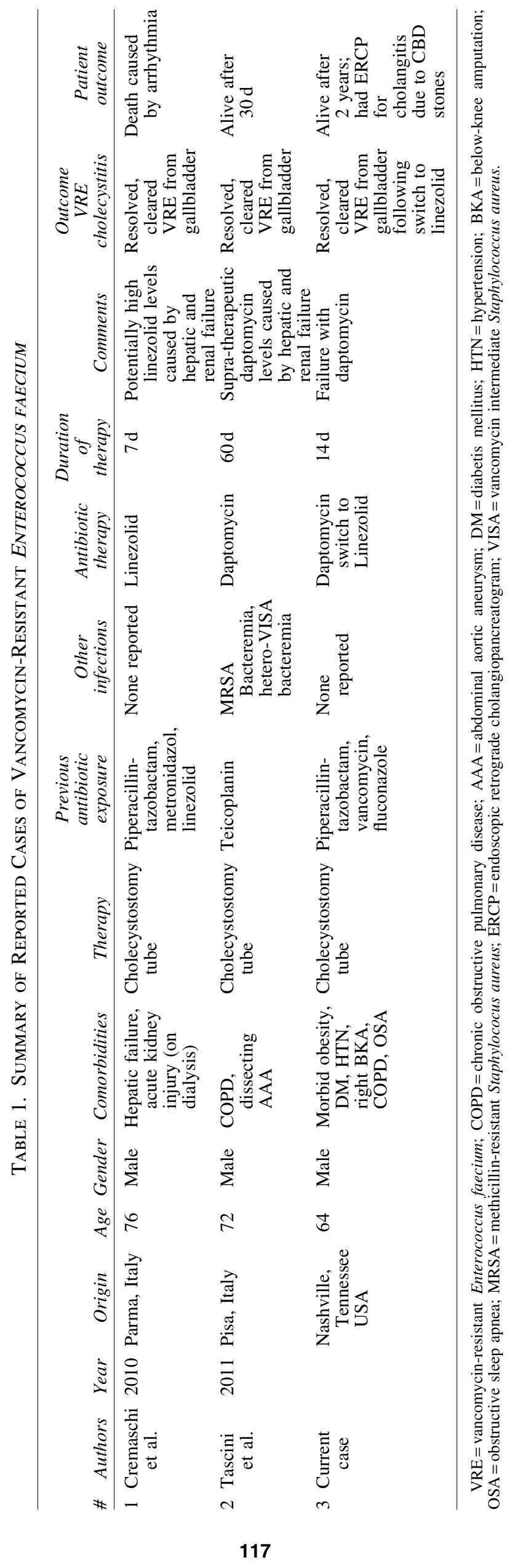


cholangiography through the cholecystostomy tube was performed (Fig. 2) and correct drain placement was confirmed; the cystic duct was patent, the common bile duct was of normal caliber with unremarkable intrahepatic biliary system; contrast promptly entered the duodenum. The patient was discharged to a nursing care facility on hospital day 19. Antibiotics were continued for a total of $14 \mathrm{~d}$ from the initiation of daptomycin. Cholecystectomy was planned after substantial weight loss and control of the comorbid conditions. The patient was ultimately discharged home from a nursing facility but did not lose any weight. The cholecystostomy tube was accidently removed after six months and not replaced because the patient had no signs of cholecystitis. Two years later, he had an episode of cholangitis and an ERCP was done with extraction of multiple common bile duct stones. Because of the high operative risk no cholecystectomy was done.

\section{Discussion}

We report the first case of VRE acute cholecystitis in the United States. This pathogen should be considered in patients with this condition who had previous exposure to antibiotics. In light of the recent emergence of various multi-drug-resistant organisms such as community-aquired methicillin-resistant Staphylococcus aureus (CA-MRSA), extended spectrum $\beta$ lactamase (ESBL), and Klebsiella pneumoniae carbapenemase (KPC)-producing gram-negative bacilli and VRE, it seems reasonable to recommend culturing bile from drains at least in subsets of patients. This may add critical information not only for optimizing treatment for the patient but also in order to protect health care workers and other patients.

Residents of long-term care facilities appear to be a reservoir for VRE and other multi-drug-resistant organisms. In a prospective cohort study, $45 \%$ of patients admitted to an acute care hospital from a long-term care facility had rectal colonization with VRE [12]; risk factors included prior use of antibiotics and the presence of a decubitus ulcer. Identification of VRE in biliary tract infection is rare with the important exception of liver transplant recipients with cholangiopathy [13]. This patient population is prone to become colonized with VRE; in fact VRE is an important pathogen causing cholangitis in this patient population [7].

The decision to manage this patient with a cholecystostomy tube and not perform surgery was based on his morbid obesity and his extensive comorbidities. Temporizing acute cholecystitis by drainage has been shown to be safe and feasible particularly in patient with extensive peri-operative risk [14]. In fact it is unclear if cholecystectomy is necessary and it has not been determined when this should be done $[15,16]$. Antibiotics alone may be able to treat acute cholecystitis appropriately [1], however, our patient most likely would not have received an agent active against VRE if no microbiologic data were available. As placement of a cholecystostomy tube is a relative simple procedure, this strategy may be more widely used in the setting of acute cholecystitis.

Our literature search revealed two additional cases of VRE cholecystitis; both were identified in Italy [10,11]. Table 1 summarizes our case and the two published cases. All three patients were critically ill and all had been exposed to broadspectrum antibiotics prior to VRE cholecystitis. Both patients from Italy had renal and hepatic failure and therefore high levels of antibiotics were achieved. In contrast, our patient was morbidly obese and neither for linezolid nor daptomycin good pharmacologic data in this patient population are available. Linezolid shows good penetration into bile $[10,13]$; in the reported patients with renal and liver failure daptomycin was recovered from bile, however, this was associated with high plasma levels. We had no capacity to do pharmacologic studies in our patient; in addition we have no reported minimum inhibitory concentrations (MICs) for our VRE isolate. The fact that daptomycin could not clear the pathogen from bile made us speculate that inadequate drug levels were achieved. In contrast linezolid was able to clear VRE from the patient's bile.

To summarize, VRE may cause acute cholecystitis in subsets of patients and best antimicrobial treatment has not yet been defined. In patients with high peri-operative risk, a cholecystostomy tube is a safe strategy to treat acute cholecystitis with the advantage of enabling microbiologic diagnosis and directed therapy of the causing organism.

\section{Author Disclosure Statement}

No competing financial interests exist.

\section{References}

1. Kanafani ZA, Khalife N, Kanj SS, et al. Antibiotic use in acute cholecystitis: Practice patterns in the absence of evidence-based guidelines. J Infect 2005;51:128-34.

2. Zehetner J, Degnera E, Olasky J, et al. Percutaneous cholecystostomy versus laparoscopic cholecystectomy in patients with acute cholecystitis and failed conservative management: A matched-pair analysis. Surg Laparosc Endosc Percutan Tech 2014;24:523-527.

3. Gosain S, Bonatti H, Smith L, et al. Gallbladder stent placement for prevention of cholecystitis in patients receiving covered metal stent for malignant obstructive jaundice: A feasibility study. Dig Dis Sci 2010;55:2406-2411.

4. Mukaiya M, Hirata K, Katsuramaki T, et al. Isolated bacteria and susceptibilities to antimicrobial agents in biliary infections. Hepatogastroenterology 2005;52:686-690.

5. Westphal JF, Brogard JM. Biliary tract infections: A guide to drug treatment. Drugs 1999;57:81-91.

6. Chou YY, Lin TY, Lin JC, et al. Vancomycin-resistant enterococcal bacteremia: Comparison of clinical features and outcome between Enterococcus faecium and Enterococcus faecalis. J Microbiol Immunol Infect 2008;41:124-129.

7. Newell KA, Millis JM, Arnow PM, et al. Incidence and outcome of infection by vancomycin-resistant Enterococcus following orthotopic liver transplantation. Transplantation 1998;65:439-442.

8. Metzger R, Bonatti H, Sawyer R. Future trends in the treatment of serious Gram-positive infections. Drugs Today (Barc) 2009;45:33-45.

9. Bassetti M, Merelli M, Temperoni C, Astilean A. New antibiotics for bad bugs: Where are we? Ann Clin Microbiol Antimicrob 2013;12:22.

10. Cremaschi E, Maggiore U, Maccari C, et al. Linezolid levels in a patient with biliary tract sepsis, severe hepatic failure and acute kidney injury on sustained low-efficiency dialysis (SLED). Minerva Anestesiol Nov;76(11):961-4.

11. Tascini C, Di Paolo A, Polillo M, Ferrari M, Lambelet P, Danesi R, et al. Case report of a successful treatment of methicillin-resistant Staphylococcus aureus (MRSA) bacteremia and MRSA/vancomycin-resistant Enterococcus faecium cholecystitis by daptomycin. Antimicrob Agents Chemother 2011;55:2458-2459 
12. Elizaga ML, Weinstein RA, Hayden MK. Patients in longterm care facilities: A reservoir for vancomycin-resistant enterococci. Clin Infect Dis 2002;34:441-446.

13. Pea F, Viale $P$, Lugano $M$, et al. Biliary penetration and pharmacodynamic exposure of linezolid in liver transplant patients. J Antimicrob Chemother 2009;63:167-169.

14. Atar E, Bachar GN, Berlin S, et al. Percutaneous cholecystostomy in critically ill patients with acute cholecystitis: Complications and late outcome. Clin Radiol 2014;69:e247-252.

15. Griniatsos J, Petrou A, Pappas P, et al. Percutaneous cholecystostomy without interval cholecystectomy as definitive treatment of acute cholecystitis in elderly and critically ill patients. South Med J 2008;101:586-590.

16. Zerem E, Omerovic S. Can percutaneous cholecystostomy be a definitive management for acute cholecystitis in high-risk patients? Surg Laparosc Endosc Percutan Tech 2014;24:187-191.

Address correspondence to:

Dr. Hugo Bonatti University of Maryland Community Medical Group Surgical Care 500 Cadmus Lane, Suite 205 Easton, MD, 21601

E-mail: Hugo.bonatti@dr.com

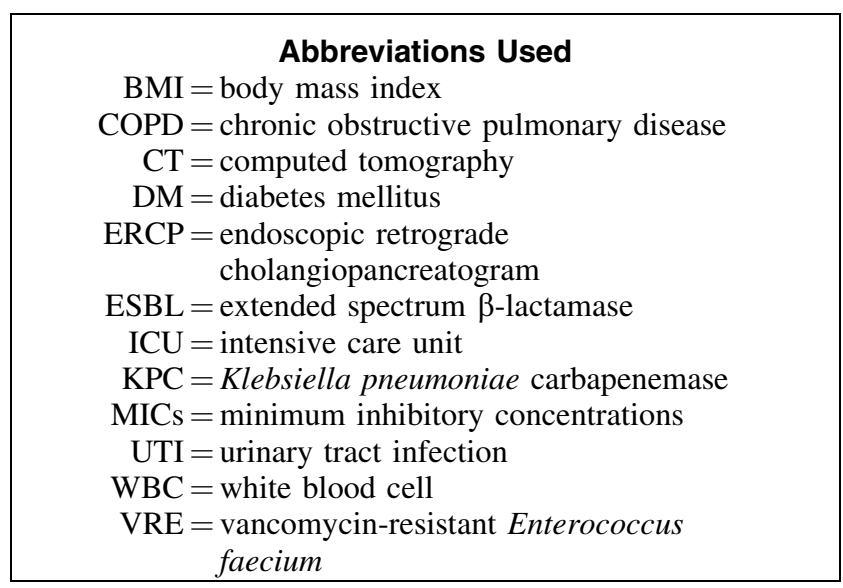

Cite this article as: Bonatti $\mathrm{H}$, Tierney $\mathrm{J}$, Kanaya E, Crislip Z, Tarpley J, May A (2016) Acute cholecystitis due to vancomycin-resistant Enterococcus faecium in a morbidly obese patient with multiple co-morbidities. Surgical Infections Case Reports 1:1, 115-119, DOI: 10.1089/crsi.2016.0027 